\title{
Neue Wirkprinzipien gegen SLE in der klinischen Testung
}

Schon länger sind keine neuen Optionen für die Therapie des systemischen Lupus erythematodes (SLE) hinzugekommen. Daher wurden die Ergebnisse von zwei Studien mit Spannung erwartet - zu Baricitinib beim SLE und zu Abatacept bei der Lupus-Nephritis.

\begin{abstract}
- Die Inhibition des JanusKinasen(JAK)-Signalweges durch den JAK-Inhibitor Baricitinib wäre für Patienten interessant, weil es eine einfach anzuwendende Therapieform mit nur einmal täglicher oraler Gabe wäre. Klinisch ist dieser Ansatz attraktiv, weil er auf die andere Achse der Pathophysiologie abzielt. Er setzt nicht an der BZelle als Produzent von Antikörpern an, sondern an den Januskinasen als Vermittler der Signaltransduktion von Zytokinen wie Interferonen und Interleukinen $(2,12$ und 23$)$.
\end{abstract}

》) Die renale Funktion (eGFR) verschlechterte sich unter Abatacept nicht

\section{Baricitinib}

Die auf dem EULAR in Amsterdam vorgestellte Studie fand eine im Vergleich zu Placebo signifikant höhere Wirksamkeit bei insgesamt akzeptabler Verträglichkeit. Die 314 untersuchten SLE-Patienten hatten zusätzlich zu einer stabilen Standard-Immunsuppression einmal täglich entweder 2 oder 4 mg Baricitinib oder Placebo erhalten. Primärer Endpunkt war die Reduktion von Arthritis und Hautsymptomatik gemäß SLEDAI-2 L (Systemic Lupus Erythematosus Disease Activity Index 2000). Unter $4 \mathrm{mg}$ Baricitinib wurde er von signifikant mehr Patien-

Quelle: Dr. Wiebke Kathmann, SpringerMedizin.de

\section{Weitere Endpunkte}

Die Überlegenheit des JAK-Inhibitors spiegelte sich auch in weiteren Endpunkten wie SRI-4(Systemic Lupus Erythematosus Responder Index)Response (64 versus $48 \%, p<0,05$ ), Reduktion der Flarerate (SFI, SELENASLEDAI Flare Index), Erreichen eines Zustands der niedrigen Krankheitsaktivität (LLDAS, Lupus Low Disease Activity State) und Reduktion der Zahl der schmerzhaften Gelenke (SJC) wider. Unter beiden Baricitinib-Dosierungen kam es allerdings aufgrund von Nebenwirkungen häufiger zum Therapieabbruch, wie in der Abstract session „Opening Plenary Abstract session“ zu hören war (OP0019).

) Unter 4 mg Baricitinib erreichten signifikant mehr Patienten den primären Endpunkt als unter Placebo

\section{Abatacept}

Das Ergebnis der in Amsterdam vorgestellten Phase 3-Studie zu Abatacept bei Lupus-Nephritis Klasse III und IV überraschte, hatte sich der selektive T-Zell-Co-Stimulations-Modulator doch in einer Phase 2-Studie als wirksam und gut verträglich erwiesen. Nun wurde der primäre Endpunkt nicht erreicht, der als komplettes renales Ansprechen nach einem Jahr definiert war und sich aus vier Aspekten zusammensetzte: erhaltener glomerulärer Filtrationsrate, einem Protein zu Kreatinin Verhältnis im Urin (UPCR) von $\leq 0,5$, dem Fehlen von Erythrozytenzylindern und einer Prednisondosis von $\leq 10 \mathrm{mg} / \mathrm{d}$.

Eingeschlossen wurden $406 \mathrm{~Pa}$ tienten mit aktiver proliferativer Lupus Nephritis, wie im Rahmen der Abstract session „Present and future treatments for SLE, Sjögren and APS" (OP0253) berichtet wurde. Zusätzlich zu einer Hintergrundtherapie aus Mycophenolat und Glukokortikoiden erhielten sie über 24 Monate entweder Placebo oder Abatacept intravenös, zunächst für drei Monate in der Dosierung von $30 \mathrm{mg} / \mathrm{kg}$, dann in der Dosierung $10 \mathrm{mg} / \mathrm{kg}$ alle vier Wochen.

Klinisch relevante Verbesserungen Auch wenn der primäre Endpunkt nicht erreicht wurde, fanden sich dennoch klinisch relevante Verbesserungen gegenüber Placebo: Die Proteinurie besserte sich deutlich schneller (Tag 85) und nachhaltiger, was zu einem tendenziell früheren und länger anhaltenden kompletten Ansprechen führte. Die renale Funktion (eGFR) verschlechterte sich unter Abatacept nicht. Zudem gingen Komplementverbrauch und Spiegel von anti-DoppelstrangDNA-Antikörpern stärker zurück. Das Sicherheitsprofil war akzeptabel.

rheuma plus $2018 \cdot 17: 118$ https://doi.org/10.1007/s12688018-0197-8

○) Springer-Verlag GmbH Austria, ein Teil von Springer Nature 2018 